\title{
Two-step homogenization of asphalt mixtures
}

\author{
R. Valenta \& M. Šejnoha \\ Czech Technical University in Prague, Faculty of Civil Engineering, \\ Department of Mechanics, Thákurova 7, 16629 Prague 6, Czech Republic
}

\begin{abstract}
A novel approach to the prediction of the nonlinear macroscopic response of asphalt mixtures is presented. It combines the well established first order homogenization method and a concept of so called statistically equivalent periodic unit cell that was only recently proposed. Such a unit cell allows us to take into account a real microstructure of an asphalt mixture when searching for the estimates of nonlinear macroscopic response. To that end, both the finite element and the Fast Fourier Transform methods are examined. To enhance feasibility of the solution of the underlying nonlinear problem, a two-step homogenization procedure is proposed. Here, the effective material properties are first found for a mastic asphalt, a composite consisting of a bitumen matrix and a fraction of small particles. These properties are then introduced in place of the matrix in actual unit cells. Several numerical examples are presented to show applicability of the proposed approach.

Keywords: asphalt mixture, statistically equivalent periodic unit cell, homogenization, Fast Fourier Transform, finite element method.
\end{abstract}

\section{Introduction}

The main objective of this contribution is to provide estimates of the effective properties of asphalt mixtures shown in Fig. 1. A computational analysis taking into account all geometrical details of a two-phase microstructure (stone aggregates bonded to a bitumen matrix) would, however, be prohibitively expensive. The search for an efficient computational scheme is therefore needed.

A relatively simple, yet reliable and efficient approach is proposed. It relies on a popular uncoupled multi-scale homogenization technique taking advantage of 


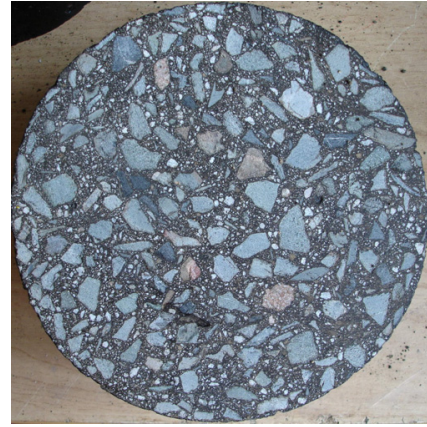

(a)

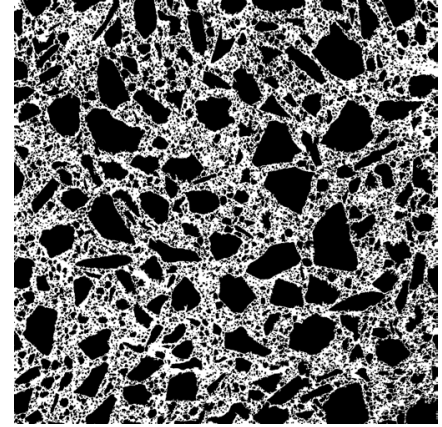

(b)

Figure 1: (a) A real microstructure of an asphalt mixture, (b) A binary image of an RVE taken from the original color image.

the concept of a statistically equivalent periodic unit cell. To arrive at the desired effective properties then requires completion of the following steps:

- Preparing a binary image of a real microstructure such as the one plotted in Fig. 1(b). This step, however, goes beyond the present scope and will not be addressed herein. Instead, the interesting reader is referred to [1].

- Construction of a suitable Representative Volume Element (RVE) from real microstructures to enhance computational efficiency. This subject receives our attention in Section 2.

- Formulation of a statistically equivalent periodic unit cell (SEPUC). This issue will be briefly addressed in Section 3.1.

- Evaluation of the non-linear effective properties for the periodic unit cell. This topic is discussed in Section 3.2.

\section{Derivation of effective elastic moduli from the original microstructure}

This section is concerned with the derivation of the effective elastic properties from the original microstructure. It will serve as a point of departure for generating the statistically equivalent periodic unit cells on the one hand and as a source of data to check the quality of the results obtained for artificial microstructures (SEPUCs) in Section 3 on the other.

\subsection{Construction of a representative volume element}

Section 2.2 introduces a very robust numerical technique for the evaluation of the effective elastic properties directly from the binary images of real microstructures. Nevertheless, it still proves computationally very exhausting if applied to original images with all details such as the one in Fig. 1(b). In [1], a procedure eliminating 


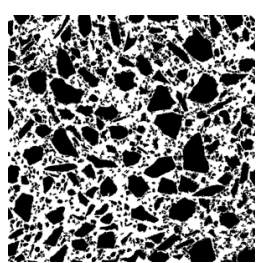

(a)

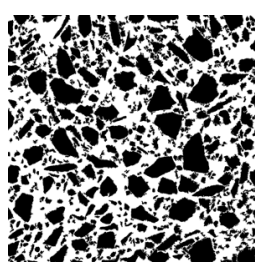

(b)

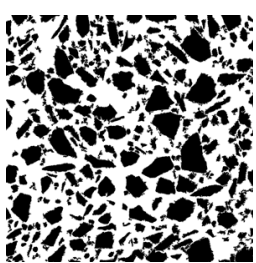

(c)

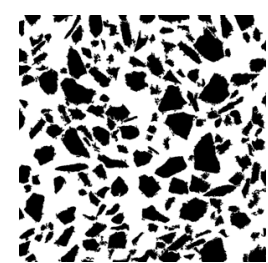

(d)

Figure 2: Examples of binary images of the original microstructure after eliminating stone fragments smaller than (in area): (a) $150 \mathrm{px}$, (b) $300 \mathrm{px}$, (c) 600 px, (d) 1200 px.

small fragments of stones up to a certain size to reduce the complexity of the original microstructure was proposed. Several such RVEs are evident in Fig. 2.

To make sure that the eliminated small stones will still contribute to the overall stiffness, a two step homogenization approach is needed. In such a case, the original bitumen is replaced by a mastic asphalt that accounts for the removed small stone fragments. The homogenized effective properties of the mastic are derived from an independent homogenization step discussed in Section 2.3. A suitable numerical technique based on classical micromechanics to carry out the computational steps is outlined next.

\subsection{Fast Fourier Transform (FFT)}

In the absence of inelastic effects, the classical micromechanical schemes can be identified with the following local constitutive relation

$$
\sigma(x)=\mathbf{L}(x) \varepsilon(x)=\mathbf{L}^{0} \varepsilon(x)+\tau(x),
$$

where $\boldsymbol{\tau}(\boldsymbol{x})=\left(\mathbf{L}(\boldsymbol{x})-\mathbf{L}^{0}\right) \boldsymbol{\varepsilon}(\boldsymbol{x})$ is the so called polarization stress and $\mathbf{L}^{0}$ represents the stiffness matrix of a certain homogeneous, generally anisotropic, reference medium. If the polarization stress is known, the local strain field $\boldsymbol{\varepsilon}(\boldsymbol{x})$ can be obtained via Green's function $\Gamma^{0}$ for a given reference medium in the form, see e.g. [2],

$$
\boldsymbol{\varepsilon}(\boldsymbol{x})=\boldsymbol{E}-\int_{\Omega} \Gamma^{0}\left(\boldsymbol{x}-\boldsymbol{x}^{\prime}\right) \boldsymbol{\tau}\left(\boldsymbol{x}^{\prime}\right) \mathrm{d} \boldsymbol{x}^{\prime} .
$$

After inserting the polarization stress $\boldsymbol{\tau}(\boldsymbol{x})$ into Eq. (2) we obtain the so called periodic Lippmann Schwinger integral equation for a given reference medium as

$$
\boldsymbol{\varepsilon}(\boldsymbol{x})+\int_{\Omega} \Gamma^{0}\left(\boldsymbol{x}-\boldsymbol{x}^{\prime}\right)\left(\mathbf{L}\left(\boldsymbol{x}^{\prime}\right)-\mathbf{L}^{0}\right) \boldsymbol{\varepsilon}\left(\boldsymbol{x}^{\prime}\right) \mathrm{d} \boldsymbol{x}^{\prime}=\boldsymbol{E} .
$$

This equation can be solved by the following iterative procedure

$$
\boldsymbol{\varepsilon}^{k+1}(\boldsymbol{x})=\boldsymbol{E}-\int_{\Omega} \boldsymbol{\Gamma}^{0}\left(\boldsymbol{x}-\boldsymbol{x}^{\prime}\right)\left(\mathbf{L}\left(\boldsymbol{x}^{\prime}\right)-\mathbf{L}^{0}\right) \boldsymbol{\varepsilon}^{k}\left(\boldsymbol{x}^{\prime}\right) \mathrm{d} \boldsymbol{x}^{\prime} .
$$



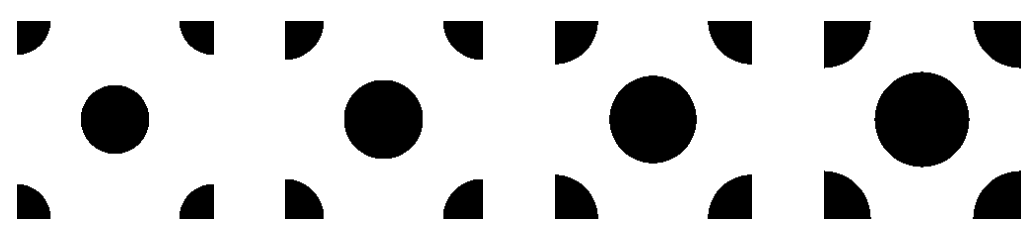
(a) $A_{s}=0.08 A$
(b) $A_{s}=0.12 A$
(c) $A_{s}=0.16 A$
(d) $A_{s}=0.21 A$

Figure 3: PUC of mastic asphalt containing stone fragments smaller than (in area):

(a) $150 \mathrm{px}$, (b) $300 \mathrm{px}$, (c) $600 \mathrm{px}$, (d) $1200 \mathrm{px}$.

Table 1: Effective properties of mastic asphalt.

\begin{tabular}{|l|c|c|c|c|c|c|}
\hline & Stone & Bitumen & PUC (a) & PUC (b) & PUC (c) & PUC (d) \\
\hline$\left.E_{\text {eff }} \mathrm{MPa}\right]$ & 30000 & 3000 & 4110 & 4465 & 4817 & 5197 \\
$v_{\text {eff }}[-]$ & 0.20 & 0.35 & 0.31 & 0.31 & 0.32 & 0.32 \\
\hline
\end{tabular}

Typically, the FFT (or rather its discrete version when dealing directly with binary images) is employed to solve the above equation. Details on actual numerical implementation can be found in [2].

\subsection{Results}

The effective elastic moduli derived for individual images in Fig. 2 using the FFT method are plotted as columns with a checkerboard pattern in Fig. 4. Clearly, the eliminated fragments of stones have a considerable impact on the predicted effective properties. To avoid this unpleasant feature a two-step homogenization approach is needed. Hence, the effective properties of a mastic asphalt composed of a bitumen matrix and a corresponding fraction of the eliminated stones are derived first assuming a simplified micromechanical model (a hexagonal periodic cell (PUC)) in Fig. 3. The results are stored in Table 1. The effective properties of the mastic asphalt are then used in place of the original bitumen when treating individual RVEs in Fig. 2. The final results appear as dash-pattern columns in Fig. 4.

It is obvious that a significant improvement can be reached when using modified bitumen matrices. These results confirm the validity of this approach and support the use of coarse RVEs in the derivation of a statistically equivalent periodic unit cell presented next. 


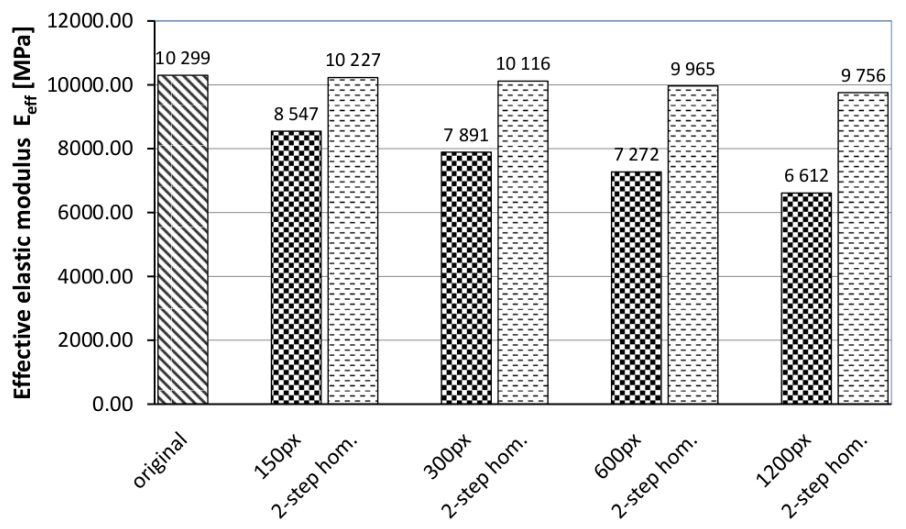

Figure 4: Effective elastic modulus derived from images in Fig. 2.

\section{Derivation of effective elastic moduli from the artificial microstructure}

Although considerable savings in computational time can be achieved with coarse RVEs, the analysis employing original microstructures still presents a significant challenge particularly in view of a large scale nonlinear analysis of multi-layered rodes. A possible route that further contributes to the efficiency of the computational analysis is discussed next in conjunction with a statistically equivalent periodic unit cell [3].

\subsection{Construction of a statistically equivalent periodic unit cell}

Suppose that the original microstructure can be replaced by a certain artificial periodic unit cell that, from the microstructure point of view, statistically resembles the real material system in terms of, e.g. the two-point probability function. Such a unit cell can be defined by the following parameters: number of aggregates having elliptical shape, size, position, orientation and aspect ratio of the axes

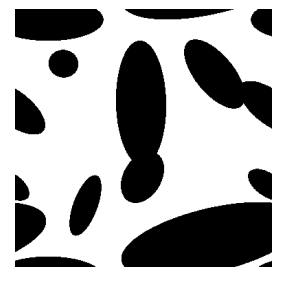

(a)

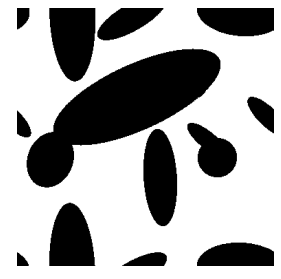

(b)

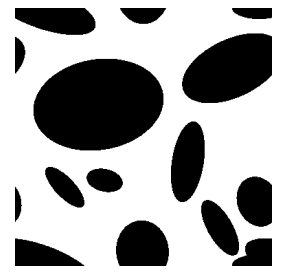

(c)

Figure 5: Examples of SEPUCs corresponding to a binary image in Fig. 2(d): (a) SEPUC 6, (b) SEPUC 43, (c) SEPUC 37. 


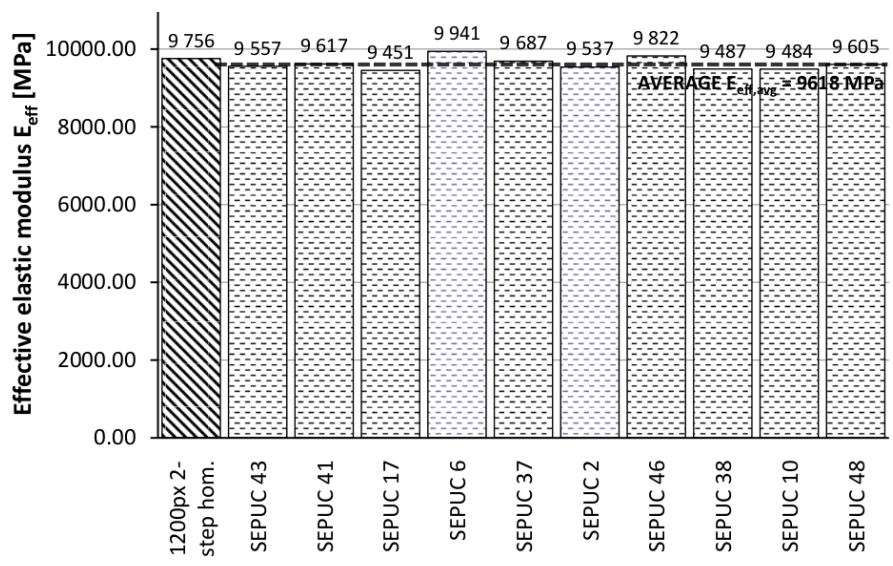

Figure 6: Effective elastic modulus derived from SEPUCs in Fig. 5.

of individual ellipses. The size of stones is derived based on the cumulative distribution function [1]. For example, if 10 stones are selected for a PUC then the smallest stone corresponds to an average size of $10 \%$ of the smallest stones determined from the cumulative distribution function. The next stone then reflects the size of the subsequent $10 \%$ stones, etc. Examples of such unit cells are depicted in Fig. 5.

These unit cells were derived by matching the two-point probability function of the original microstructure, Fig. 2(d), and the SEPUC. The underlying optimization problem was solved with the help of the evolutionary algorithm GRADE [5]. See also [3, 4] for other applications of genetic algorithm based solution strategies.

As typical of genetic algorithms based optimization procedures, each run results in a unique SEPUC with a slightly different arrangement of stones, see Fig. 5. It therefore remains to be confirmed that all cells provide the "same" macroscopic response. Note that for the sake of efficiency the target microstructure in Fig. 2(d) with modified bitumen properties (the last column in Table 1) was used when generating artificial periodic microstructures. The corresponding effective properties are represented by the first column in Fig. 6. The remaining columns refer to the effective elastic modulus for the selected SEPUCs (100 such cells were generated). Although slightly different in their geometrical details they all provide nearly the same macroscopic response almost identical to the original microstructure.

In most practical applications, however, the mastic asphalt is typically loaded beyond the elastic regime. The issue, whether the geometrical invariance of SEPUCs outlasts even for a non-linear response should be examined. For simplicity, we limit our attention to finite element simulations presented in the framework of the first-order homogenization theory. The theoretical formulation is outlined next. The corresponding results are discussed in Section 3.3. 


\subsection{First order homogenization in view of finite element analysis}

Consider an RVE given in terms of a SEPUC in Fig. 2. The stepping stone when deriving the effective material properties of a composite aggregate is provided by Hill's lemma, which for compatible strain and equilibrated stress fields reads, see [3] for further details,

$$
\langle\boldsymbol{\sigma}(\boldsymbol{x}): \boldsymbol{\epsilon}(\boldsymbol{x})\rangle=\langle\boldsymbol{\sigma}(\boldsymbol{x})\rangle:\langle\boldsymbol{\epsilon}(\boldsymbol{x})\rangle=\boldsymbol{\Sigma}: \boldsymbol{E},
$$

where $\boldsymbol{\Sigma}$ and $\boldsymbol{E}$ represent the prescribed overall uniform stress and strain fields, respectively. The macroscopic constitutive relations are provided by

$$
\boldsymbol{\Sigma}=\langle\boldsymbol{\sigma}(\boldsymbol{x})\rangle=\langle\mathbf{L}(\boldsymbol{x})(\boldsymbol{\varepsilon}(\boldsymbol{x})-\boldsymbol{\mu}(\boldsymbol{x}))\rangle=\mathbf{L}(\boldsymbol{E}-\boldsymbol{\mu}),
$$

where $\boldsymbol{\mu}(\boldsymbol{x})$ and $\boldsymbol{\mu}$ are the local and macroscopic eigenstrain vectors, representing the non-linear viscoelastic effects developed in the matrix phase. These are derived from the generalized Leonov model, which is assumed here to represent the behavior of the mastic asphalt. Details regarding the implementation of the generalized Leonov model are provided in [6]. Next, with the help of Eqs. (5) and (6), we write the average Lagrange variational principle in the form

$$
\begin{aligned}
\mathbf{W}(\boldsymbol{E}) & =\frac{1}{2 V} \int_{V} \boldsymbol{\varepsilon}(\boldsymbol{u}(\boldsymbol{x}))^{\mathrm{T}} \mathrm{L}(\boldsymbol{x})(\boldsymbol{\varepsilon}(\boldsymbol{u}(\boldsymbol{x}))-\boldsymbol{\mu}(\boldsymbol{x})) \mathrm{d} \boldsymbol{x} \\
& \leq \frac{1}{2 V} \int_{V} \boldsymbol{\epsilon}(\boldsymbol{v}(\boldsymbol{x}))^{\top} \mathrm{L}(\boldsymbol{x})(\boldsymbol{\varepsilon}(\boldsymbol{v}(\boldsymbol{x}))-\boldsymbol{\mu}(\boldsymbol{x})) \mathrm{d} \boldsymbol{x},
\end{aligned}
$$

where $\boldsymbol{u}(\boldsymbol{x})$ represents the solution of the above problem and $\boldsymbol{v}(\boldsymbol{x})$ is any kinematically admissible displacement field. To introduce a kinematically admissible field we split the local displacements $\boldsymbol{v}(\boldsymbol{x})$ into average and fluctuation parts such that

$$
\boldsymbol{v}(\boldsymbol{x})=\boldsymbol{E} \boldsymbol{x}+\boldsymbol{v}^{*}(\boldsymbol{x}),
$$

where the fluctuation part $\boldsymbol{v}^{*}$ enters Eq. (8) as a consequence of the presence of heterogeneities and has to disappear upon volume averaging. This condition is met for any periodic displacement field with the period equal to the size of the unit cell under consideration.

Finally, after taking the variation of Eq. (7) with respect to $\boldsymbol{u}(\boldsymbol{x})$ we arrive, in conjunction with standard Finite Element discretization, at the system of equilibrium equations in the form

$$
\mathbf{K} \boldsymbol{u}^{*}=\boldsymbol{f},
$$

where

$$
\begin{array}{rlrl}
\mathbf{K} & =\sum_{e} \mathbf{K}^{e} & \text { where } & \mathbf{K}^{e}=\frac{1}{\Omega} \int_{e} \mathbf{B}^{\mathrm{T}} \mathbf{L}^{e} \mathbf{B} \mathrm{d} \Omega^{e}, \\
\boldsymbol{f}=\sum_{e} \boldsymbol{f}^{e} & \text { where } & \boldsymbol{f}^{e}=-\frac{1}{\Omega} \int_{e} \mathbf{B}^{\mathrm{T}} \mathbf{L}^{e}(\boldsymbol{E}+\boldsymbol{\mu}(\boldsymbol{x})) \mathrm{d} \Omega^{e},
\end{array}
$$




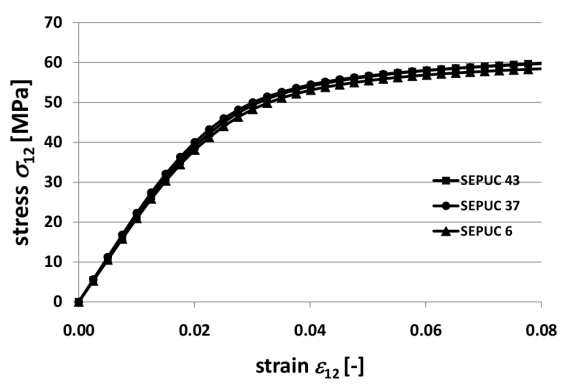

(a)

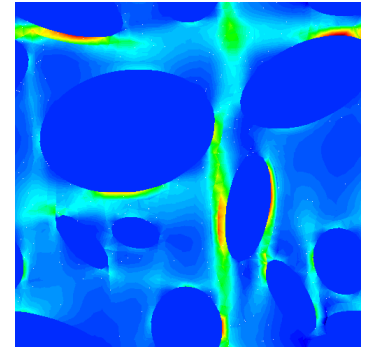

(b)

Figure 7: Macroscopic non-linear response from SEPUCs in Fig. 5: (a) Stressstrain curve, (b) Variation of equivalent local strain.

where $\Omega$ is the cell area. Owing to the non-linear effects, this system of equations must be solved incrementally.

\subsection{Results}

As an example we considered a problem of a unit cell loaded by the prescribed constant rate of the macroscopic shear strain. The elastic parameters of the matrix phase (the entire stiffness matrix) were those used for the matrix phase of the RVE in Fig. 2(d), recall also the last column in Table 1. Since the experimental program for the derivation of non-linear properties of various bitumen asphalts is currently under way, we adopted (noticing a similar elastic behavior) the same non-linear material properties for the generalized Leonov model that we used for the polymeric matrix in [1].

The resulting macroscopic stress-strain curves are plotted in Fig. 7(a) for the three SEPUCs in Fig. 3. The results are rather appealing, since any of the cells can actually be used, if we are not interested in the particular distribution of local fields within the cell. To compare these results with the response derived from original RVEs requires an extension of the FFT method to non-linear viscoelasticity. This is the subject of our present effort.

\section{Conclusion}

This paper presents a summary of the preliminary work on asphalt mixtures. It concentrates on the morphological description of actual microstructure on the one hand and on the effect of the elimination of small stones from the original complex microstructure on the other. While the total number of stones eliminated is relatively high their volume fraction when compared to the total volume of stones is negligible. The effective properties are, however, considerably affected. To compensate for the loss of microstructural details due to stone elimination needed in the optimization process the two-step homogenization procedure is 
considered. The results presented in Sections 2.3 and 3.3 confirm applicability of this approach in both the elastic and inelastic regimes.

\section{Acknowledgement}

The financial support of the Ministry of Education, Youth and Sports, project No. 1M0579 is gratefully acknowledged.

\section{References}

[1] Valenta, R. \& Šejnoha, M., Construction of statistically equivalent periodic unit cell of asphalt mixture. Proceedings of the Eleventh International Conference on Civil, Structural and Environmental Engineering Computing, ed. B.H.V. Topping, Civil-Comp Press, 2007. On CD-ROM.

[2] Moulinec, H. \& Suquet, P., Micromechanics of materials: From numerical simulations to predictive models. 3rd National Congress on Computational Mechanics, ed. J.K. N. Avaras, pp. 93-100, 1999.

[3] Zeman, J. \& Šejnoha, M., Numerical evaluation of effective properties of graphite fiber tow impregnated by polymer matrix. Journal of the Mechanics and Physics of Solids, 49(1), pp. 69-90, 2001.

[4] Matouš, K., Lepš, M., Zeman, J. \& Šejnoha, M., Applying genetic algorithms to selected topics commonly encountered in engineering practice. Computer Methods in Applied Mechanics and Engineering, 190(13-14), pp. 1629-1650, 2000.

[5] Hrstka, O. \& Kučerová, A., Improvements of real coded genetic algorithms based on differential operators preventing the premature convergence. Advances in Engineering Software, 35(3-4), pp. 237-246, 2004.

[6] Šejnoha, M., Valenta, R. \& Zeman, J., Nonlinear viscoelastic analysis of statistically homogeneous random composites. International Journal for Multiscale Computational Engineering, 2(4), pp. 645-673, 2004. 